VoL. $62(2000)$ [171-173]

\title{
Semilinear elliptic equations with critical nonlinearities
}

\author{
ELLIOT TONKES
}

In recent times, the following family of problems has engaged the interest of those working with variational methods in partial differential equations:

$$
-\Delta u=\lambda g(x) u+f(x, u)
$$

where $\Delta$ is the Laplacian operator and $\lambda$ is a real parameter. Different domains of definition have invoked interest and various boundary conditions have been considered. In this thesis, the unbounded domain $\mathbf{R}^{N}$ is analysed. Solutions are sought in the Sobolev space $D^{1,2}\left(\mathbf{R}^{N}\right)$.

In the thesis, issues concerning existence are investigated, and this class of problems acts as a test bed for new methods. The majority of the thesis is concerned with semilinear equations, denoted so because nonlinearities are confined to functions of $u$ in the $f(x, u)$ term. In the final chapter, a quasilinear equation is investigated, which involves an inherently nonlinear operator, the $\mathrm{N}$-Laplacian.

The primary analysis technique corresponds with the modern paradigm for problems in a variational setting. Once the Euler-Lagrange equations have been converted to a critical point problem concerning the $C^{1}$ functional $I$, the following two step method is applied:

(1) develop a Palais-Smale sequence $\left(I\left(u_{n}\right) \rightarrow c\right.$ and $\left.I^{\prime}\left(u_{n}\right) \rightarrow 0\right)$ from the geometry of the functional and

(2) from the topology of level sets, confirm that Palais-Smale sequences at the appropriate levels converge.

This second step is termed the Palais-Smale condition at a level $c$, and initially appears restrictive.

The pioneering work of Brézis and Nirenberg in their seminal paper [2] confirmed that this methodology was indeed adequate, and appropriate for dissemination of problems of a wide variety. This paper treated problems involving the critical Sobolev exponent, $2^{*}$. In accordance with the Sobolev embedding theorem, the inclusion of a critical exponent means that the variational formulation is at its very limit.

Received 31st January, 2000

Thesis submitted to The University of Queensland, April 1999. Degree approved, August 1999. Supervisor: Dr J. Chabrowski.

Copyright Clearance Centre, Inc. Serial-fee code: 0004-9727/00 \$A2.00+0.00. 
This thesis focuses on critical nonlinearities with sign indefinite characteristics. The sign-indefinite nature of nonlinearities becomes apparent in the strong indefiniteness of the corresponding functional.

The thesis uses minimax methods which are based on the Ekeland variational principle. Minimax techniques characterise a critical value $c$ of the functional $I$ by following the recipe

$$
c=\inf _{A \in S} \max _{u \in A} I(u) .
$$

Selecting the class $S$ distinguishes the various minimax methods, which include the mountain pass theorem, linking theorems and the fountain theorems.

In chapter 2 the nonlinearity $f(x, u)$ in (1) takes the form of two competing convex nonlinearities; $f(x, u)=k(x)|u|^{q-2} u-h(x)|u|^{p-2} u$. In the setting of bounded domains, this problem has been analysed in [1]. Competition between the nonlinearities occurs because a relation concerning the supports of $k(x)$ and $h(x)$ is enforced. In lieu of imposing a compactness condition, two integrability conditions are introduced which allow the exponent $p$ to exceed the critical Sobolev exponent.

Existence of a solution around the first positive and first negative eigenvalues is assured, and depending on how the nonlinearity interacts with the first eigenfunction, two solutions may exist beyond the first resonance. The results are expanded by invoking Rabinowitz' global bifurcation theorem.

In Chapter 3, the perturbation $f(x, u)$ is set to the form $h(x)|u|^{2^{*}-2} u$ and the associated functional is a limiting case of the Palais-Smale condition. Because the embedding of the underlying space $D^{1,2}\left(\mathbf{R}^{N}\right)$ into the Lebesgue space $L^{2^{*}}\left(\mathbf{R}^{N}\right)$ is only continuous and not compact, Palais-Smale sequences may evaporate.

This chapter extends the work by Drábek and Huang [3] by using an alternative method. The concentration-compactness principles are invoked to establish a PalaisSmale condition below and above the first resonance. Below resonance, a mountain pass solution is obtained. Provided that a standard compactness condition is confirmed, the geometry of the functional is verified to elicit a mountain pass and local minimum solution in a right neighbourhood of the principal eigenvalue.

The case of dimension three poses an interesting dilemma. Local bifurcation results guarantee that a solution exists in a neighbourhood of the principal eigenvalue. However, the estimates which proved valid for dimensions beyond four appear inadequate to handle the three dimensional case.

Extending this investigation into Chapter 4, the issue of multiplicity is considered for small $\lambda$. Requiring $\lambda$ strictly positive means that the constrained minimisation is prevented from concentrating to a delta-distribution.

The problem is tackled in two different ways. The first involves a delicate analysis on Nehari manifolds. By applying careful analysis, the manifolds are shown to possess multiple 'pockets', corresponding to the number of maximal regions of the nonlinearity. 
Each pocket donates a solution. The cleaner way involves Lusternik-Schnirelmann category, borrowing some of the earlier estimates. For both of these investigations, as the perturbation term diminishes, solutions are found to concentrate to delta distributions.

In Chapter 5, the nonlinearity assumes the form of competing concave and convex terms: $f(x, u)=k(x)|u|^{q-2} u-h(x)|u|^{p-2} u$. The geometry of the functional drastically alters when $q$ falls below 2, and the problem warrants application of fountain theorems.

Firstly, a new fountain theorem and a new dual fountain theorem are formulated. Essentially, the new results allow a broader class of functionals by identifying and extracting a positive definite subspace which does not interfere with the critical levels.

If $k(x) \geqslant 0$ and $p<2^{*}$, then a dual fountain theorem provides an infinitude of solutions. If $h(x) \leqslant 0$, then the fountain theorem yields an unbounded sequence of solutions. If both weighting functions are sign indefinite, then two solutions may exist.

In case of a critical nonlinearity, if $k(x)$ and $h(x)$ are both nonnegative, then an infinite number of solutions is established. When $h(x)$ is indefinite in sign, but $k(x)$ is nonnegative, then solutions are only identified when $k(x)$ is sufficiently small.

Chapter 6 investigates a quasilinear elliptic equation involving the $N$-Laplacian in a bounded domain of $\mathbf{R}^{N}$ with a nonlinearity on the limit of the variational formulation. A different type of embedding is required involving exponential functions instead of polynomial. This chapter extends the work in [4] to a nonhomogeneous problem. Nontrivial solutions are located by local minimisation and by a mountain pass theorem, but a PalaisSmale condition is not invoked. Consequently, it is a nontrivial exercise to distinguish the solutions. Provided that the perturbation to the problem is positive, solutions are verified to be positive.

\section{REFERENCES}

[1] S. Alama and G. Tarantello, 'Elliptic problems with nonlinearities indefinite in sign', $J$. Funct. Anal. 141 (1996), 159-215.

[2] H. Brézis and L. Nirenberg, 'Positive solutions of nonlinear elliptic equations involving critical Sobolev exponents', Comm. Pure Appl. Math. 36 (1983), 437-477.

[3] P. Drábek and Y.X. Huang, 'Bifurcation problems for the $p$-Laplacian in $\mathbf{R}^{N}$, Thans. Amer. Math. Soc. 349 (1997), 171-188.

[4] J.M.B. do Ó, 'Semilinear Dirichlet problems for the N-Laplacian in $\mathbf{R}^{N}$ with nonlinearities in the critical growth range', Differential Integral Equations 9 (1996), 967-979.

School of Information Technology

Bond University Qld 4229

Australia

e-mail: etonkes@bond.edu.au 